\title{
Reimbursement Challenges in Europe for Innovative Therapies
}

\author{
Jenifer Ehreth* \\ Health Economics, University of Washington and Principal, International Market Access, Paris France
}

${ }^{\star}$ Corresponding author: Jenifer Ehreth PhD, MHA, Associate Professor emeritus Health Economics, University of Washington and Principal, International Market Access, Paris France

Received: March 23, 2019; Accepted: April 03, 2019; Published: April 05, 2019;

The thinking about the role of health care systems has been evolving over the last ten years such that there is more recognition of the importance of healthy populations on overall wealth and security [1]. At the European level and in WHO studies have shown that the positive return on investment in health systems is between $50 \%$ and $250 \%$ [2]. At the same time with the exploding advances in understanding how the body works, innovative therapies are proliferating at an ever so rapid pace [3]. What these innovations have in common that was not the case in the past is that they are more focused. Their patient populations are smaller. They are more efficient. That is to say those innovations a generation ago were targeting large populations (Block Buster drugs) with diseases for which we had a significant understanding at that time. Today as scientific understanding is digging deeper into the chemistry of the body, the challenges for health care systems have focused on the rising costs and limited budgets.

The responsibility at the European level is to make sure that new therapies are safe. Unlike in the US where the FDA is responsible for safety, they are also in some cases, responsible for reimbursement. In Europe, there is a clear distinction between these roles. Reimbursement is a Member State responsibility. To talk about reimbursement challenges immediately devolves into 27 different health care systems. So what commonalities can be used to address the way Reimbursement Authorities are taking on these challenges without becoming too bogged down into individual health care systems? This article will assess the strategies for pricing, early access, budget controls and cost-effectiveness.

\section{Pricing}

While there is a range of approaches to pricing from initially allowing free pricing as in Germany, governmental negotiation on pricing as in France, prices pegged to categories of medications as in Spain to prices determined by cost-effective algorithms as in the UK. The challenges facing Reimbursement Authorities are to identify a 'fair' price, given the pharmaceutical industry's investment in creating innovative therapies. Any meaningful discussion of the impact on pricing of access to medications or the overall health of the population is complicated by the fact that within each Member State, pricing policies are specific to where the treatment is provided (inpatient, outpatient, etc.) and often by region. Nevertheless, there is no evidence that one strategy leads to better health than another strategy. While prices do vary by country, going back to the initial premise that access to innovative treatment has a positive effect on society; it is not yet clear to what extent the price level contributes or takes away from overall societal wellbeing. That is, when does the price get so high that the benefit is not justified?

\section{Early Access}

Early access programs are available throughout Europe but the intent and foci vary greatly. The rationale for these programs is to assure that patients receive treatments that are specifically life-saving during the Regulatory (EMA level) and Reimbursement (Member State level) processes that can take from months to years. The criteria and payment for these programs range from fully funded small populations with exceptional circumstances such as in Italy to broader patient groups in France and only partially funded or not funded as in the UK [4]. Some programs become available as soon as the new drug is being reviewed at the European level whereas others wait until approval is given but the Member State has yet to finish its deliberative process. Some of the key considerations of manufacturers are how the program may effect pricing and what additional safety studies may be required. There is general acceptance that these programs do provide a needed bridge that allows access to innovation that would not have been possible without their existence.

\section{Budget Controls}

Given the conflicting forces of improved health benefits and rising costs, the mechanism Member States are focusing on are ways to manage their budgets. One of the limitations with this effort is that budgets are divided into categories that are self-contained. That is, the health care budget is reviewed and those in charge are responsible irrespective of other budgets. Hence, positive results from expenditures in health are not monetised and applied to non-health budgets. Worse yet, within the health system, the various budgets are also independent. A classic example is when an innovative medication results in patients avoiding hospitalisations. The drug budget goes up and the hospital budget goes down in reality but is not captured in a meaningful way.

Strategies to account for cross-budget effects are beginning to emerge. One important improvement has been in the move in all 
Member States to capitated hospital budgets. There are various names for this but they are adaptations of the Diagnostic Related Grouping that was adopted in the US for Medicare hospital payments in the 1980s. At least in hospitals, the medication costs are not independently budgeted. If an innovation results in a shorter length of hospital stay, the hospital is able to benefit in the short run.

Non-hospital budget control strategies have been focused on pharmacy constraints such as requiring generic substitution. Depending on the details of the policy, this could impact the willingness of manufacturers of innovative medications to offer access.

As yet, no budgetary control system has been able to integrate the budget impact of improved health from innovative therapies on the wealth and economic success of the country.

\section{Cost-effectiveness}

Another strategy for improved reimbursement decisions has been the advent of economic algorithms that try to capture the costs and benefit at the same time to arrive at a single ratio to be used in decision making. There is strong conceptual support for this in economic theory. This was introduced over 20 years ago in the UK and has been adapted in several other Member States. The concept is to compare the costs to a common health outcome. Since health outcomes are not common, the most widely used created outcome is the Adjusted Life Years Saved. This ratio simplifies comparisons across disparate therapies. Without going into the mechanics of this approach, this approach presents the following issues for innovative therapies.

First is the pragmatic access to all costs over the lifetime of the public (patients, families, workplaces, etc.) affected by the therapy. While direct therapeutic costs are often in claims processing databases, other costs are not. Next is the significant shortfall in measuring the elements of the outcomes. Without going into the extensive literature on these current shortfalls, there has been an emerging area of research in Real World Data to address some of these concerns. This field is in its infancy but the future may lead to improved measurement of health outcomes. Finally and potentially more importantly is the question of: 'What is a sufficient ratio to allow reimbursement of the innovation?' Here, economic theory cannot help. There is no yardstick to determine acceptability. The UK has set ratios between $20 \mathrm{k} £$ and $30 \mathrm{k} £$ but there is no scientific support for this. Worse yet, the ratios generated for innovative therapies for small populations fall far outside of this yardstick leading countries using economic algorithms as decision making guidelines to rethink their usefulness.

\section{Conclusion}

Hopefully innovative therapies will continue to proliferate and the health and economic security of the public will benefit from them. At the same time, reimbursement policies need to evolve the take into consideration the new reality of the marvellous new world we are living in.

\section{References}

1. McKee L, Suhrcke M, Nolte E, Lessof S, Figueras J, et al (2009) Health systems, health, and wealth : a European perspective. The Lancet 373 : 349-351.
2. Suhrcke M, Rocco L, McKee M (2007) Health : a vital investment for economic development in eastern Europe and central Asia. Copenhagen : WHO Regional Office for Europe on behalf of the European Observatory on Health systems and Policies, 2007.

3. Godman B, Bucsics A, Vella Bonanno P, Oortwijn W, Rothe C, et al (2018) Barriers for access to new medicines: Searching for the balance between rising costs and limited budgets. Fronties in Public Health 328.

4. Urbinati D, Masetti L, Toumi M (2012) Early Access Programmes (EAPs) : Review of European system, ISPOR 15th Annual European Congress, Berlin, Germany.

\section{Citation:}

Ehreth J (2019) Reimbursement Challenges in Europe for Innovative Therapies. $J$ Pharmacol Pharm Res Volume 2 (2): 1-2. 